\title{
ASPECTOS SOCIODEMOGRÁFICOS E OBSTÉTRICOS DA MORBIDADE MATERNA GRAVE ${ }^{1}$
}

\author{
SOCIODEMOGRAPHIC AND OBSTETRIC ASPECTS \\ OF SEVERE MATERNAL MORBIDITY
}

\section{ASPECTOS SOCIODEMOGRÁFICOS Y OBSTÉTRICOS DE LA MORBILIDAD MATERNA GRAVE}

\author{
Camila Marcelino Loureiro* \\ Cláudia Rios Cataño ** \\ LAILla TORRICELLI $^{* * *}$ \\ Juliana Cristina dos Santos Monteiro ${ }^{* * * *}$ \\ FlÁVIA A. GOMES-SPONHOLZ ${ }^{* * * *}$
}

\begin{abstract}
RESUMO
Objetivo: Identificar os aspectos sociodemográficos, obstétricos e de saúde de mulheres com experiência de morbidade materna grave em um hospital de nível terciário. Material e método: Estudo descritivo, transversal, de base hospitalar realizado em 78 mulheres. A coleta de dados realizou-se por meio de entrevistas estruturadas e revisão de prontuários e os dados foram analisados mediante estatística descritiva. Resultados: Das participantes $24,4 \%$ foram gestantes e $75,6 \%$ puérperas, a idade média foi de 28,8 anos. $35,9 \%$ foram primigestas e os principais diagnósticos foram as síndromes hipertensivas, 83,3 e 16,7\% outras morbidades. Destaca-se que $15,4 \%$ das participantes atendiam a mais de um critério durante o tempo de internação e 73,0\% tiveram seis ou mais consultas de pré-natal. Conclusão: As síndromes hipertensivas foram as primeiras causas de morbidade materna grave, e ressalta-se a assistência pré-natal prestada. Os resultados fornecem implicações para o financiamento de mecanismos de assistência às mulheres que sofreram os eventos da morbidade materna grave associados aos aspectos obstétricos e sociodemográficos das mesmas. Existe a necessidade de caracterizar e monitorar a morbidade materna grave na atenção obstétrica para desenvolver ações de promoção e melhorar os cuidados de saúde prestados a essas mulheres. O conhecimento dos principais diagnósticos apresentados por mulheres com morbidade materna grave são importantes para a prática dos enfermeiros que atuam nessa área, pois permite instrumentalizar mudanças no modelo de assistência à mulher e sua família, com vistas a que se atinja a maternidade segura em nosso país.
\end{abstract}

\footnotetext{
${ }^{1}$ Resultados extraídos parcialmente da tese de doutorado de Cláudia Rios Cataño intitulada: Depressão, ansiedade e transtorno de estresse pós-traumático em mulheres que vivenciaram um episódio de morbidade materna grave. Programa de Pós-Graduação em Enfermagem em Saúde Pública da Escola de Enfermagem de Ribeirão Preto (EERP) da Universidade de São Paulo (USP), Brasil, 2011.

* Enfermeira. Graduada pela Escola de Enfermagem de Ribeirão Preto da Universidade de São Paulo, Brasil. Email: camilalloureiro@hotmail.com

** Obstetriz. Doutora pelo programa de Pós-Graduação Enfermagem em Saúde Pública da Escola de Enfermagem de Ribeirão Preto da Universidade de São Paulo, Ribeirão Preto - SP, Brasil. Email: rcclaudinha@hotmail.com

${ }^{* * *}$ Enfermeira. Graduada pela Escola de Enfermagem de Ribeirão Preto da Universidade de São Paulo, Ribeirão Preto SP, Brasil. Email: lailla.t@hotmail.com

${ }^{* * * *}$ Enfermeira Obstetra. Doutora. Escola de Enfermagem de Ribeirão Preto da Universidade de São Paulo, Ribeirão Preto - SP, Brasil. Email: jumonte@eerp.usp.br

${ }^{* * * * *}$ Enfermeira Obstetra. Doutora. Livre Docente. Escola de Enfermagem de Ribeirão Preto da Universidade de São Paulo, Ribeirão Preto - SP, Brasil. Email: flagomes@eerp.usp.br
} 
Palavras chave: Morbidade, complicações na gravidez, gestação de alto risco, fatores de risco, enfermagem obstétrica.

\begin{abstract}
Objective: Identify sociodemographic, obstetric and health aspects of women who experienced severe maternal morbidity in a tertiary care hospital. Method: A descriptive study, cross-sectional and hospital-based involving 78 women. Data collection was carried out through structured interviews and review of medical records and data were analyzed using descriptive statistics. Results: $24.4 \%$ of participants were pregnant and $75.6 \%$ were in the postpartum period, the average age was 28.8 years. $35.9 \%$ were primiparous and primary diagnoses were hypertensive disorders, $83.3 \%$ and $16.7 \%$ other morbidities. It is noteworthy that $15.4 \%$ of participants met more than one criterion during the time of admission and $73.0 \%$ had six or more prenatal consultations. Conclusion: Hypertensive syndromes were the first causes of severe maternal morbidity, and prenatal care provided was highlighted. The results provide implications for the funding of mechanisms to assist women who have suffered from severe maternal morbidity events associated with obstetric and sociodemographic aspects. There is a need to characterize and monitor severe maternal morbidity in obstetric care to develop actions to promote and improve the health care provided to these women. Knowledge of the mains diagnosis presented by women with severe maternal morbidity are important for the practice of nurses working in this area, because it allows for the implementation of changes in the care model for women and their families, with a view to the attainment of safe motherhood in our country.
\end{abstract}

Key words: Morbidity, pregnancy complications, high-risk pregnancy, risk factors, obstetric nursing.

\title{
RESUMEN
}

Objetivo: Identificar los aspectos sociodemográficos, obstétricos y de salud en mujeres con experiencia de morbilidad materna grave en un hospital de nivel terciario. Material y método: Estudio descriptivo, transversal, realizado en hospital con 78 mujeres. La recolección de datos se realizó mediante entrevistas estructuradas y revisión de historias clínicas, los datos fueron analizados mediante estadística descriptiva. Resultados: De las participantes $24,4 \%$ fueron gestantes y $75,6 \%$ puérperas, la edad media fue de 28,8 años, el $35,9 \%$ fueron primigestas. Los principales diagnósticos fueron: síndromes hipertensivos (83,3\%), otras morbilidades 16,7\%; se destaca que el 15,4\% de las participantes presentaban más de un criterio durante el tiempo de internación y el $73 \%$ tuvo seis o más consultas de prenatal. Conclusión: Los síndromes hipertensivos fueron las primeras causas de morbilidad materna grave, y se resalta la asistencia prenatal prestada. Los resultados proporcionan implicaciones para la financiación de mecanismos de asistencia a las mujeres que sufrieron los eventos de morbilidad materna grave asociados a los aspectos obstétricos y sóciodemográficos de las mismas. Existe la necesidad de caracterizar y monitorizar la morbilidad materna grave durante la atención obstétrica para desarrollar acciones de promoción y mejorar los cuidados de salud prestados a estas mujeres. El conocimiento del perfil de diagnósticos presentados en las mujeres con morbilidad materna severa es importante para la práctica de los enfermeros que trabajan en esta área, ya que permite dotar a los cambios en el modelo de atención de la mujer y su familia, con miras al logro de una maternidad segura en nuestro país.

Palabras clave: Morbilidad, complicaciones del embarazo, embarazo de alto riesgo, factores de riesgo, enfermería obstétrica.

Fecha recepción: 29/04/15 Fecha aceptación: 30/12/16 


\section{INTRODUÇÃO}

Atualmente, mais de meio milhão de mulheres morrem todos os dias, no mundo todo, por causas relacionadas ao ciclo gravídicopuerperal (1). Destas mortes, aproximadamente 99\% ocorrem em países em desenvolvimento, sendo um evento raro em países desenvolvidos. De acordo com os dados fornecidos pelo Programa das Nações Unidas para o Desenvolvimento, em 2000 as taxas de mortalidade materna nas regiões em desenvolvimento (440/100.000 nascidos vivos) foram 20 vezes maiores que nas regiões desenvolvidas (20/100.000 nascidos vivos) (2).

No Brasil, a razão de mortalidade materna apresentada oficialmente é de 64,8/100.000 nascidos vivos (nv) (3), com piores indicadores nas regiões Norte $(77,8 / 100.000 \mathrm{nv})$ e Nordeste (80,8/100.000 nv) (4). No entanto, a real magnitude da mortalidade materna permanece desconhecida, com estimativas que variam de 38 a 87/100.000 nascidos vivos, entretanto sabe-se que $15 \%$ das gestações apresentam complicações durante o ciclo grávido-puerperal e que parte destas evoluirão para óbito $(5,6)$, sendo fundamental a avaliação da morbidade materna com o objetivo de desenvolver estratégias para prevenção do desfecho mais grave. Ainda, pela ocorrência mais frequente da morbidade em relação à morte materna, considera-se que este estudo ofereça informações consistentes, uma vez que as próprias mulheres sobreviventes podem ser uma valiosa fonte de informação (7).

Ao longo dos anos, classificações distintas foram utilizadas para descrever as complicações severas do ciclo gravídico-puerperal, como morbidade materna, morbidade extrema, near miss e morbidade obstétrica (5). Recentemente a Organização Mundial da Saúde (OMS) estabeleceu a definição de morbidade materna, sendo o termo morbidade extremamente grave/near miss como a "uma mulher que quase morreu, mas que sobreviveu a uma complicação que ocorreu durante a gestação, parto ou dentro de 42 dias do término da gravidez" (8).

No presente estudo utilizou-se a definição de morbidade materna grave proposta pela Organização Mundial de Saúde (9), que considera um evento de morbidade severa em mulheres durante a gestação, parto ou pós-parto, classificada como uma condição potencial de ameaça a vida, incluindo, hemorragia, hipertensão ou outras alterações sistêmicas. O estudo da morbidade materna grave em um hospital de referência nacional possibilita instrumentalizar mudanças no modelo de assistência à mulher e sua família, para poder atingir a maternidade segura no país. Além disso, contribui na redução de barreiras que as impedem de acessar os serviços de saúde e na adoção de medidas preventivas da morbidade materna grave/near miss em diversos níveis, ao identificar suas características e condições clínicas mais frequentes, importantes para o enfrentamento da morbimortalidade materna no Brasil.

Progredindo para a investigação dos fatores associados à morbidade materna, alguns estudos apontam como principais fatores de risco a idade materna, a cor da pele não-branca, a exclusão social, a presença de hipertensão, o antecedente de aborto e fatores obstétricos diretos como hemorragia pós-parto, indução do parto e a realização de cesariana (10). Entre o grande número de fatores associados que são investigados em pesquisas sobre morbidade materna, além da idade acima dos 35 anos, permaneceram como fatores de risco o relato de hipertensão prévia à gestação, ter antecedente de aborto e ter feito menos consultas de pré-natal do que o mínimo recomendado pelo Ministério da Saúde. Em diferentes estudos, as principais causas de morbidade materna foram pré-eclâmpsia e eclâmpsia, hemorragia no período pós-parto, sepse puerperal, aborto e trabalho de parto obstruído. No entanto, a incidência de cada um destes eventos varia entre os países e mesmo entre regiões de um 
mesmo país e o resultado final, está associado aos recursos disponíveis (11).

O monitoramento de complicações obstétricas tem se mostrado relevante para avaliar tendências na qualidade da assistência em estudos em países em desenvolvimento (10). Considera-se que este é um estudo que contribuirá para a construção do conhecimento em enfermagem, pois permite a identificação das situações predisponentes para a morbidade materna grave e seus desdobramentos, trazendo benefícios para as mulheres no período reprodutivo e suas famílias. Além de esta pesquisa estar fundamentada na humanização, gênero, maternidade segura, direitos reprodutivos e a integralidade do cuidado.

Este estudo têm como hipóteses (a) as síndromes hipertensivas e transtornos hemorrágicos serem as principais causas para as mulheres desenvolverem morbidade materna grave; (b) e os principais fatores de risco da morbidade materna serem a idade materna avançada, a exclusão social, hipertensão prévia à gestação, o antecedente de aborto e ter realizado menos consultas de pré-natal do que o mínimo recomendado pelo Ministério da Saúde. Nessa perspectiva o estudo teve como objetivo identificar os aspectos sociodemográficos, obstétricos e de saúde de mulheres com experiência de morbidade materna grave.

\section{MATERIAL E MÉTODO}

Trata-se de um estudo descritivo e transversal. Realizado em um hospital de referência de nível terciário de atenção em um município do interior do estado de São Paulo.

Participaram deste estudo, mulheres que preenchiam os critérios de morbidade materna grave (9), hospitalizadas no serviço de obstetrícia, entre os meses de junho a agosto de 2011. O recrutamento das participantes foi realizado mediante visita diária de alunas de graduação e pós-graduação à unidade de internação clínica e revisão de todos os prontuários de mulheres com idade entre 10 e 49 anos e que estavam internadas, por no mínimo, 48 horas, para identificar aquelas que preenchiam os critérios de morbidade materna grave.

Escolheu-se um horário específico para realizar as entrevistas, tendo em vista não coincidir com a visita médica, não atrapalhar a rotina da equipe de enfermagem ou a da paciente. A realização das entrevistas foi, em média, duas horas por dia.

Foram utilizados dois instrumentos, sendo um questionário com respostas estruturadas para a obtenção de informações sociodemográficas e psicossociais; e outro elaborado com base nas experiências prévias dos pesquisadores e cotejado com a literatura, para a obtenção de informações relacionadas ao histórico obstétrico e a gestação atual, relativas ao evento de morbidade materna grave.

Para a coleta de dados considerou-se as variáveis sociodemográficas (idade, escolaridade, atividade remunerada, lugar de procedência e estado civil), variáveis obstétricas (consultas de pré-natal, tipo de parto - dado coletado no pós-parto, intercorrências na gestação, parto e/ou no puerpério e amamentação) e variáveis do histórico de saúde (realização de consultas médicas previas à gravidez, uso de medicamentos, uso de substâncias químicas e procura por serviços de saúde).

Os dados foram armazenados na planilha eletrônica Excel Mac 2011 e validados mediante dupla digitação. Para a análise utilizou-se o programa SPSS, versão 16.0. Realizou-se a análise mediante estatística descritiva (distribuição de frequências, medidas de tendência central e variabilidade).

O estudo foi aprovado por um Comitê de Ética em Pesquisa, cadastrado na Comissão Nacional de Ética em Pesquisa (CONEP), sob o número de protocolo 1305/2011. Todas as participantes assinaram o Termo de Consentimento Livre e Esclarecido e ficaram com uma via em sua posse. 


\section{RESULTADOS}

Nos meses de junho a agosto de 2011 foram hospitalizadas 534 mulheres na unidade de internação clínica, das quais 78 mulheres preenchiam os critérios de morbidade materna grave, representando $14,6 \%$ do total de internações ocorridas nesses meses. Dentre as 78 participantes, $27(34,6 \%)$ foram hospitalizadas para a resolução da gravidez e 51 $(65,4 \%)$ para tratamento clínico. Cinquenta e nove $(75,6 \%)$ estavam na primeira internação. A média de internações foi de $1,38 \mathrm{com}$ desvio-padrão de 0,81 .

\section{Perfil sociodemográfico}

Do total das participantes, 19 (24,4\%) eram gestantes e $59(75,6 \%)$ puérperas. Em relação à escolaridade, encontrou-se a maior frequência de mulheres no ensino médio 42 (53,9\%), e em relação ao estado civil, 30 estavam em união estável $(38,5 \%)$. Quanto à procedência, $47(60,3 \%)$ residiam no município em que o hospital está situado (Tabela 1 ). A idade média das mulheres do estudo foi de 28,8 anos, com desvio padrão de 7,81, a idade mínima 15 anos e a máxima de 43 anos. A respeito do número de pessoas que moravam com a participante, considerando-se a entrevistada como o primeiro integrante obteve-se o máximo de 13 pessoas na mesma residência e o mínimo de duas, com uma média de 3,79 pessoas e desvio-padrão de 3,0 .

Tabela 1. Descrição de variáveis sóciodemográficas e obstétricas das pacientes com morbidade materna grave. Ribeirão Preto, SP. 2011.

\begin{tabular}{lcc}
\hline Variáveis & $\mathrm{f}$ & $(\%)$ \\
\hline Sociodemográficas & & \\
Mulheres com morbidade materna grave & 19 & 24,4 \\
$\quad$ Gestantes & 59 & 75,6 \\
Puérperas & & \\
Escolaridade & 31 & 39,7 \\
$\quad$ Ensino fundamental & 42 & 53,9 \\
Ensino médio & 5 & 6,4 \\
Ensino superior & & \\
Estado civil & 30 & 38,5 \\
$\quad$ União estável & 28 & 35,9 \\
Casadas & 13 & 16,6 \\
Solteiras & 5 & 6,4 \\
Separadas & 2 & 2,6 \\
Viúvas & & \\
Procedência & 47 & 60,3 \\
$\quad$ Ribeirão Preto & 31 & 39,7 \\
22 cidades próximas & & \\
Obstétricas & 26 & 33,3 \\
Primíparas & 12 & 15,4 \\
Tiveram abortos & 60 & 73 \\
$\quad$ 6 consultas pré-natais & & \\
\hline
\end{tabular}




\section{Perfil obstétrico}

Dentre as 78 mulheres, a maioria era multigesta $50(64.1 \%)$, sendo que uma participante $(1,3 \%)$ estava na oitava gestação. A média foi de 2,73 gestações por participante, com desvio padrão de 1,83 , número máximo gestações 8 e mínimo de 1. Quanto ao número de partos, $26(33,3 \%)$ eram primíparas, nove $(1,5 \%)$ eram nulíparas, com média de 2,32 partos por participante, desvio-padrão de 1,8 e mediana de dois, o número máximo de partos sete e mínimo de 0 . Em relação ao número de abortos, 12 participantes (15,4\%) afirmaram terem tido abortos em sua vida, com o número máximo de três abortos.

Em relação às consultas de pré-natal, 60 mulheres $(73,0 \%)$ tiveram mais de seis consultas (Tabela 1), sendo o mínimo zero, o máximo de 18 consultas, com uma média de 8,33 consultas e desvio padrão de 4,25.

\section{Perfil de saúde}

Em relação ao histórico de saúde, investigou-se se as participantes realizavam acompanhamento médico prévio à gravidez. Os dados mostraram que 58 (74,4\%) faziam seguimento em instituições de saúde, e as especialidades médicas mais procuradas foram cardiologia e medicina geral e os principais eventos foram hipertensão arterial (40\%) e infecções das vias aéreas (15\%) (Gráfico 1).

Quanto à procura de serviço de saúde mental $19(24,4 \%)$ afirmaram ter procurado o serviço, sendo os sintomas depressivos a principal causa. Quanto ao uso de medicamentos prévios à gravidez 34 participantes (43,6\%) afirmaram utilizar medicamentos diários, sendo os anti-hipertensivos os mais utilizados (62,0\%) (Gráfico 2). Também identificou-se que $58(74,4 \%)$ eram usuárias do sistema único de saúde.

Gráfico 1. Distribuição da frequência (n) e percentual (\%) das variáveis relacionadas às razões das consultas médicas referidas pelas mulheres com morbidade materna grave.

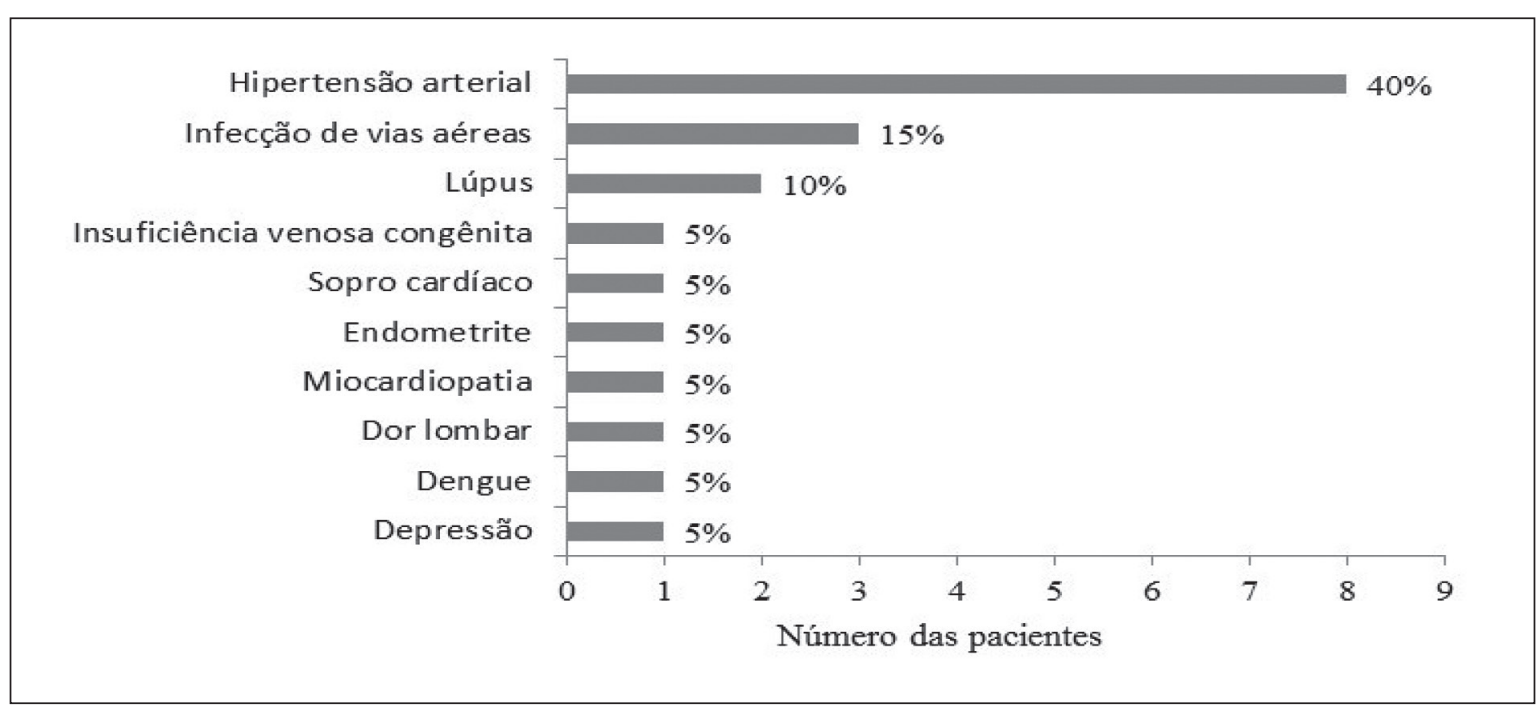


Gráfico 2. Distribuição da frequência (n) e percentual (\%) das variáveis relativas ao uso de medicamentos referidos pelas mulheres com grave morbidade materna.

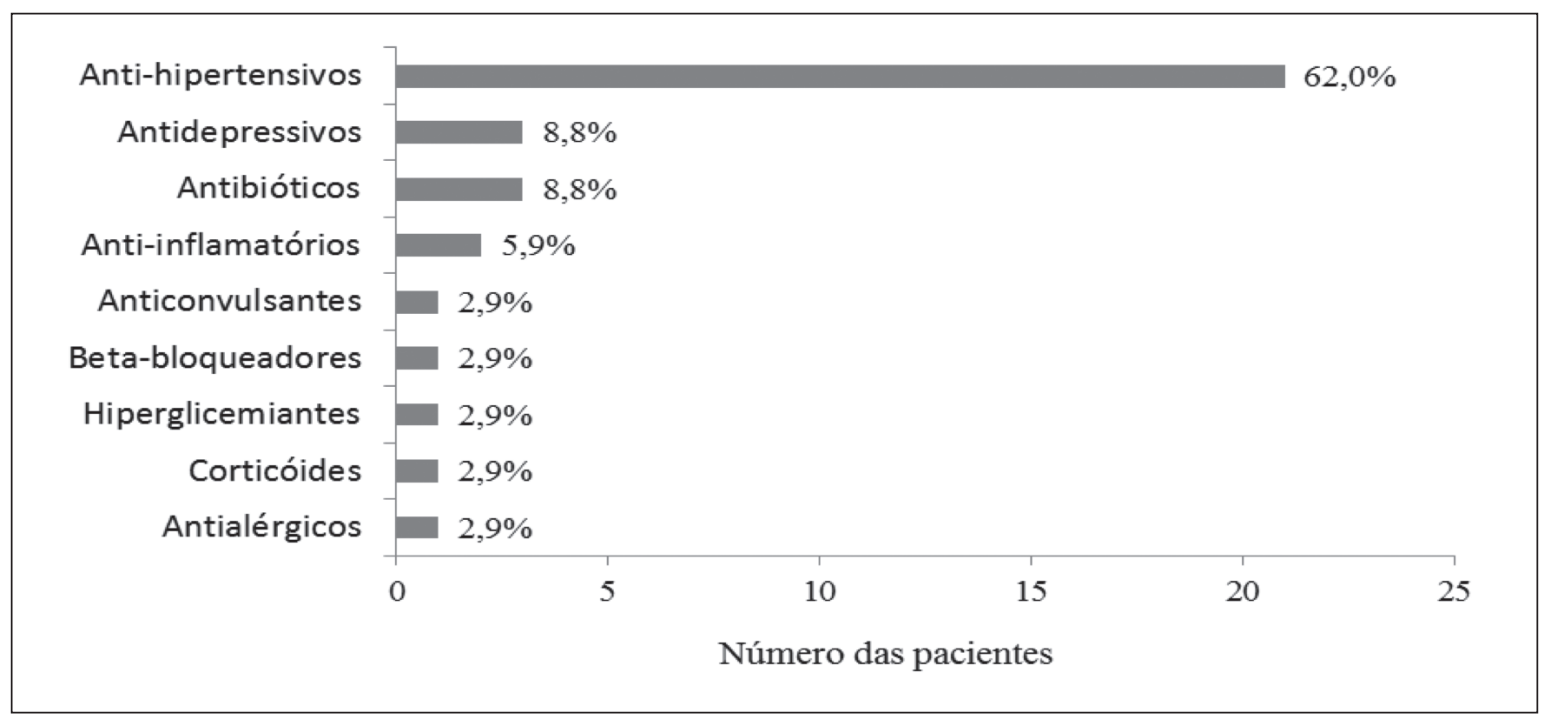

No que tange ao uso de substâncias psicoativas prévias a gestação, 16 (20,5\%) responderam afirmativamente para o uso, sendo o cigarro a principal substância. Em menor proporção, maconha, crack e cocaína.

Do total de participantes, 59 (75,6\%) estavam internadas pela primeira vez no ciclo grávido-puerperal atual e 12 (15,4\%) estavam na segunda internação. A média de internações foi de 1,38 com desvio padrão 0,81 . Quanto à unidade onde permaneceram todo o período de internação, 75 (96,2\%) foram admitidas pelo serviço de obstetrícia e três $(3,8 \%)$ pela Unidade de Terapia Intensiva (UTI). Dentre as admitidas na UTI duas $(2,6 \%)$ tiveram sepse e uma (1,3\%) apresentou três diagnósticos no momento da admissão: embolia pulmonar, coagulação intravascular disseminada e insuficiência respiratória aguda. Esta última participante foi a única a precisar de intubação orotraqueal por 24 horas. O tempo médio de permanência em UTI foi de 3,33 dias com desvio padrão de 0,58.

Quanto à morbidade materna grave, 65 participantes $(83,3 \%)$ tiveram agravos relacionados às síndromes hipertensivas, sendo pré-eclâmpsia, hipertensão grave, eclâmpsia e síndrome HELLP. Outras morbidades como os distúrbios hemorrágicos e distúrbios sistêmicos se apresentaram em 13 mulheres $(16,7 \%)$. Destacou-se que 12 participantes $(15,4 \%)$ atendiam a mais de um critério para morbidade materna grave durante o tempo de internação. A participante com maior quantidade de critérios apresentou nove morbidades na mesma internação. Em relação ao momento do ciclo grávido-puerperal em que o agravo ocorreu, sete (11,9\%) apresentaram intercorrências durante o parto, sendo a hemorragia a mais frequente em 4 $(8,0 \%)$ destas. Durante a condução do trabalho de parto encontrou-se a ocorrência $(1,3 \%)$ de rotura uterina, doença hipertensiva e problemas não especificados.

No período puerperal 12 participantes $(15,4 \%)$ apresentaram complicações; sendo as doenças hipertensivas as intercorrências mais frequentes, manifestada em seis mulheres $(7,7 \%)$, seguida por hemorragia em duas participantes $(2,6 \%)$ e em número de uma ocorrência (1,3\%) abcesso na parede vaginal, diabetes, uma paciente apresentou hipertensão arterial e diabetes e outra hipertensão arterial e hemorragia. 
Seis participantes $(7,7 \%)$ foram submetidas à transfusão de sangue, sendo que três precisaram de duas unidades, uma recebeu três unidades, uma paciente precisou de quatro unidades e uma recebeu 21 unidades, sendo uma média de 5,67, desvio padrão de 7,55 e mediana 2,5. Cinco participantes das sete $(83,3 \%)$ receberam hemácias e uma $(16,7 \%)$ recebeu hemácias, plasma e plaquetas.

\section{DISCUSSÃO E CONCLUSÃO}

Com a formulação dos Objetivos de Desenvolvimento Sustentável, em 2015, os países signatários das Nações Unidas acordaram nova agenda que reafirma o compromisso em reduzir a morbimortalidade por causas preveníveis e evitáveis, com a implementação de ações de saúde que contribuam para a garantia dos direitos humanos das mulheres (12). Com base neste compromisso, entende-se que a análise da distribuição de variáveis sociais, demográficas e de saúde das mulheres que tiveram um episódio de morbidade materna grave contribua para a adequação a nível local, das estratégias propostas pelos gestores nacionais, atendendo às necessidades de saúde da mulher e prevenindo riscos.

Quanto ao ciclo grávido-puerperal atual, dentre as mulheres que apresentaram morbidade materna grave no estudo, $24,4 \%$ eram gestantes e $75,6 \%$ puérperas. O agravo mais frequente em todo o ciclo grávido-puerperal foi referente às doenças hipertensivas; a intercorrência mais frequente durante o parto foi hemorragia e no pós-parto também a doença hipertensiva e o sangramento. Destacase que no período avaliado, não houve caso de morte materna. No estudo de Morse et al., (13) que avaliou três critérios para classificação de morbidade materna, o marcador de gravidade mais utilizado foi a pré-eclâmpsia grave, seguida de hemorragia grave.

Quanto ao perfil de mulheres com morbidade materna grave, estudo realizado pela
Organização Mundial da Saúde (14), mostrou que ter mais de 35 anos, sem parceiro e estar na primeira gestação ou ser multípara são variáveis associadas à ocorrência de morbidade materna extremamente grave/ near miss. Esses achados concordam com o presente estudo, sendo que 35\% de mulheres que apresentaram agravos tinham mais de 30 anos, seguidas por $21 \%$ de adolescentes (13). Quando a escolaridade é analisada juntamente com o tipo de parto, há associação entre mais anos estudados e a ocorrência desta morbidade (14). Estudo com desenho semelhante ao apresentado encontrou mulheres que tiveram episódio de morbidade materna grave, as quais estavam vivendo em união estável em mais de $50 \%$ dos casos e em sua primeira gestação. Na revisão da literatura científica encontrou-se diferenças nas associações das variáveis sociodemográficas e obstétricas com morbidade materna grave e extremamente grave/near miss (13).

Estudo sugere que a relação entre gravidez, doenças crônicas, morbidade e mortalidade materna pode estar aumentando (15). Fatores como sedentarismo, tabagismo, sobrecarga de responsabilidades são gerados pelo estilo de vida moderno e contribuem para que doenças crônico-degenerativas estejam entre as principais causas de morte na população feminina (16). O passado de hipertensão e de doenças crônicas foi descrito anteriormente como fator de risco para morbidade materna grave. No Brasil, estudo em UTI obstétrica descreve que condições clínicas pré-existentes estavam presentes em 19\% das pacientes (17).

Neste contexto, as mulheres em idade reprodutiva estão expostas a doenças crônicas e aos fatores de risco tais como obesidade, diabetes e colesterol (18). Dentre as participantes deste estudo, 60\% afirmaram fazer seguimento em serviços de saúde e uso de medicamentos, a maioria por hipertensão crônica antes da gravidez. As doenças cardiovasculares configuram-se a principal causa de morte em mulheres e a terceira causa de 
morte em mulheres em idade reprodutiva (16). A prevalência de hipertensão e dislipidemia é alta entre mulheres em idade reprodutiva e são os dois maiores fatores de risco para doenças cardiovasculares (18). O conhecimento destes dados tem levado estudiosos a pesquisar neste assunto e vários estudos têm apontado medidas que incluem programas de atividade física, reeducação alimentar, redução do tabagismo, como pontos principais na prevenção das doenças cardiovasculares dentre outras oportunidades de prevenção de agravos, melhorando a saúde das mulheres (18). Os agravos mentais que acometem as mulheres em idade reprodutiva necessitam ser investigados, bem como a identificação de grupos vulneráveis. Estas são estratégias apontadas por pesquisadores para que ocorra o diagnóstico precoce, prevenção de comorbidades e acompanhamento adequado no nível primário de atenção à saúde (14).

Em relação ao perfil obstétrico, no estudo observou-se predomínio de mulheres com três gestações anteriores, em média dois partos por mulher, sem abortos anteriores. Estudo realizado em uma instituição hospitalar de referência terciária para avaliar o perfil epidemiológico da morbidade materna grave e extremamente grave/near miss encontrou no perfil obstétrico de sua amostra 33,7\% de primíparas e $23,6 \%$ tiveram abortos prévios, a maioria foi incluída no estudo com média de 34,6 semanas (13). Outro estudo realizado para identificar o perfil reprodutivo de mulheres com diabetes mellitus (19) encontrou mulheres com idade média de 25,7 anos, com uma ou duas gestações anteriores e $25 \%$ haviam tido um aborto.

Em relação às consultas de pré-natal, autores apontam o acesso à assistência de qualidade ao pré-natal e parto, $\mathrm{o}$ acesso a suportes tecnológicos específicos, como a presença de unidade de terapia intensiva, ou mesmo o acesso a transfusões com a necessária rapidez deve estar ao alcance de todas as mulheres (5) colaborando para redução de complicações e das mortes maternas. Em relação ao pré-natal (20), encontraram que $40 \%$ de mulheres que atendiam aos critérios de morbidade materna grave, fizeram mais de seis consultas e aproximadamente $10 \%$ não realizaram qualquer consulta.

A hospitalização para a realização de tratamento clínico de alguma intercorrência no ciclo grávido puerperal ocorreu em $65,4 \%$ das mulheres deste estudo. Nos Estados Unidos, no início da década de 1990, o número de internações obstétricas durante a gravidez foi de 22 para cada 100 partos. Esta informação fomentou uma meta nacional de redução de internações obstétricas para no máximo 15 para cada 100 partos. No entanto, estudo atual (21) divulgou resultados de aumento progressivo de internações clínicas para tratamento de morbidade materna nos últimos dez anos, em Unidade de Terapia Intensiva no estado do Texas.

Há evidências de que a maioria dos casos de morbidade extremamente grave/near miss que ocorrem nos países em desenvolvimento chegam aos hospitais em condições críticas (17). Assegurar o acesso equitativo à atenção básica e atendimento especializado de emergência, o reconhecimento precoce e tratamento das condições maternas potencialmente fatais é fundamental para salvar vidas de mães e seus recém-nascidos $(6,22)$.

As síndromes hipertensivas complicam 5 a $10 \%$ de todas as gestações (15) e são a principal causa de morbidade materna grave e morbidade materna extremamente grave/ near miss durante o período gravídico-puerperal. A hipertensão, como entidade associada ou como causa direta de mortalidade materna, tem sido motivo de preocupação nos diferentes setores da comunidade médica durante a gravidez. As formas mais comuns de hipertensão são a pré-eclâmpsia, a hipertensão arterial crônica e a hipertensão crônica com pré-eclâmpsia superposta; sendo esta última é associada a piores resultados maternos e perinatais (23).

Estudo realizado entre 2003 e 2007 mos- 
trou que entre mulheres com critérios para morbidade materna grave ou extremamente grave/near miss, a causa mais comum de internação em UTI obstétrica foram as síndromes hipertensivas $(78,4 \%)$ onde a eclâmpsia ocorreu em 38,8\% dos casos. Segundo autores de outro estudo (24), as síndromes hipertensivas corresponderam a $62,5 \%$ dos casos em seu estudo, 10,6\% apresentaram quadros hemorrágicos, $5,8 \%$, sepse e $21,1 \%$ apresentaram complicações clínicas. A hipertensão foi causa quase exclusiva de morbidade materna grave no estudo realizado em 2008, sendo responsável por $47,5 \%$ dos casos de morbidade materna grave; e por $18,2 \%$ das morbidades extremamente graves/near miss. Outros estudos desenvolvidos no Brasil encontraram as síndromes hipertensivas como uma das causas mais comumente associadas à morbidade materna grave e à morbidade extremamente grave/near miss (13). A segunda causa mais incidente no presente estudo foi hemorragia. Estudos mostram que as complicações hemorrágicas, principalmente sangramento no período pós-parto, resultante de atonia uterina e ruptura uterina, são ocorrências frequentes e presentes nas causas de morbidade e mortalidade materna (5). A hemorragia obstétrica é a causa mais comum de mortalidade materna no mundo e a causa da mortalidade materna nos Estados Unidos que tem maior chance de prevenção $(24,25)$.

Os resultados fornecem implicações para o financiamento de mecanismos de assistência às mulheres que sofreram os eventos da morbidade materna grave associados aos aspectos obstétricos e sóciodemográficos das mesmas. As síndromes hipertensivas foram as primeiras causas de morbidade materna grave. Portanto, há necessidade da caracterização da morbidade materna grave em um hospital de nível terciário de atenção para o tratamento de quadros hipertensivos durante a gravidez. E os profissionais da saúde devem oferecer recursos que permitam o monitora- mento da atenção à saúde da mulher no ciclo grávido-puerperal, o desenvolvimento de ações de promoção, ações educativas e a qualificação da melhoria dos cuidados de saúde prestados às usuárias do serviço investigado.

Destaca-se também, a importância do acesso das mulheres à assistência pré-natal, pela possibilidade de identificar precocemente intercorrências e complicações da gravidez. Acreditamos que a investigação do histórico de saúde mental à anamnese tradicional é relevante, pois possibilita a determinação de riscos adicionais como depressão puerperal.

Profissionais de enfermagem inseridos na assistência obstétrica devem ter como alvo o reconhecimento dos riscos para morbidade materna e incorporar em sua prática clínica abordagens que contribuam para a promoção da equidade de gênero e dos direitos humanos das mulheres. Acompanhar e ou cuidar de uma mulher em situação de morbidade materna inclui respeito ao desejo de ser mãe, aos aspectos culturais e sociais, e ainda, considerar a assistência humanizada e acolhedora.

Com relação às limitações do presente estudo, podemos destacar a má qualidade do registro nos prontuários das participantes, por vezes incompletos ou insuficientes, resultando em subestimação de informações importantes. Acreditamos que estudos futuros possam investigar a relação entre a morbidade materna grave e as repercussões obstétricas e neonatais, de forma que as mulheres sejam acompanhadas prospectivamente, relacionando também com a percepção das mesmas sobre este agravo em suas vidas.

Agradecimentos: Agradeço ao CNPq (Conselho Nacional de Desenvolvimento Científico e Tecnológico) pelo financiamento do projeto e às mulheres com morbidade materna grave que aceitaram participar da pesquisa, pela paciência. 


\section{REFERÊNCIAS}

1. Collender G, Gabrysch S, Campbell OM. Reducing maternal mortality: better monitoring, indicators and benchmarks needed to improve emergency obstetric care. Research summary for policymakers. Trop Med Int Health. 2012; 17(6): 694-6.

2. Programa das Nações Unidas para o Desenvolvimento, PNUD. Saúde: Coleção de estudos temáticos sobre os objetivos de desenvolvimento do milênio. Objetivo 5: Melhorar a saúde materna. Belo Horizonte: PUC Minas. Brasil; IDHS, 2004. $120 \mathrm{p}$.

3. Brasil. Ministério da Saúde. Indicadores de mortalidade. Razão de mortalidade materna. Brasília: Ministério da Saúde; 2011.

4. Madeiro AP, Rufino AC, Lacerda É, Brasil LG. Incidence and determinants of severe maternal morbidity: a transversal study in a referral hospital in Teresina, Piaui, Brazil. BMC Pregnancy Childbirth. 2015; 15: 210.

5. Lotufo FA, Parpinelli MA, Haddad SM, Surita FG, Cecatti JG. Applying the new concept of maternal near-miss in an intensive care unit. Clinics (Sao Paulo). 2012; 67(3): 225-30.

6. Oliveira-Neto A, Parpinelli MA, Cecatti JG, Souza JP, Sousa MH. Sequential organ failure assessment score for evaluating organ failure and outcome of severe maternal morbidity in obstetric intensive care. Scientific World Journal [Internet]. 2012 [citado 30 jun 2015]:172145. Disponível em: https://www.ncbi.nlm.nih.gov/ pubmed/22454600

7. Cecatti JG, Costa ML, Haddad SM, Parpinelli MA, Souza JP, Sousa MH, et al. Network for Surveillance of Severe Maternal Morbidity: a powerful national collaboration generating data on maternal health outcomes and care. BJOG. 2016; 123(6): 946-53.
8. Say L, Souza JP, Pattinson RC, WHO working group on Maternal Mortality and Morbidity classifications. Maternal near miss-towards a standard tool for monitoring quality of maternal health care. Best Pract Res Clin Obstet Gynaecol. 2009; 23(3): 287-96.

9. World Health Organization. Evaluating the quality of care for severe pregnancy complications: the WHO near miss approach for maternal health. Department of Reproductive Health and Research. Genebra: WHO; 2011.29 p.

10. Moraes APP. Morbidade materna grave em São Luis. [Tese Doutorado em Saúde Pública]. [Belo Horizonte]: Universidade Federal de Minas Gerais; 2011. 121 p.

11. Senanayake H, Dias T, Jayawardena A. Maternal mortality and morbidity: epidemiology of intensive care admissions in pregnancy. Best Pract Res Clin Obstet Gynaecol. 2013; 27(6): 811-20.

12. Graham W, Woodd S, Byass P, Filippi V, Gon G, Virgo S, et al. Diversity and divergence: the dynamic burden of poor maternal health. Lancet. 2016; 388(10056): 2164-75.

13. Morse ML, Fonseca SC, Gottgtroy CL, Waldmann CS, Gueller E. Severe maternal morbidity and near misses in a regional reference hospital. Rev Bras Epidemiol. 2011; 14(2): 310-22.

14. Souza JP, Cecatti JG, Faundes A, Morais SS, Villar J, Carroli G, et al. Maternal near miss and maternal death in the World Health Organization's 2005 global survey on maternal and perinatal health. Bull World Health Organ. 2010; 88(2): 113-9.

15. Robbins CL, Dietz PM, Bombard J, Tregear M, Schmidt SM, Tregear SJ. Lifestyle interventions for hypertension and dyslipidemia among women of reproductive age. Prev Chronic Dis. 2011; 8(6): A123.

16. Moura EC, Santos WD, Neves AC, Schwarz E, Gomes R. Mortality in Brazil according to gender perspective, years 2000 and 2010. Rev Bras Epidemiol. 
2016; 19(2): 326-38.

17. Moraes AP, Barreto SM, Passos VM, Golino PS, Costa JA, Vasconcelos MX. Incidence and main causes of severe maternal morbidity in São Luís, Maranhão, Brazil: a longitudinal study. Sao Paulo Med J. 2011; 129(3): 146-52.

18. Barfield WD, Warner L. Preventing chronic disease in women of reproductive age: opportunities for health promotion and preventive services. Prev Chronic Dis. 2012; 9: E34.

19. Moura ERF, Evangelista DR, Damasceno AKDC. Conhecimento de mulheres com diabetes mellitus sobre cuidados pré-concepcionais e riscos materno-fetais. Revista da Escola de Enfermagem da USP, 2012. p. 22-9.

20. Vettore MV, Dias M, Domingues RM, Leal MoC. [Prenatal care and management of hypertension in pregnant women in the public healthcare system in Rio de Janeiro, Brazil]. Cad Saude Publica. 2011; 27(5): 1021-34.
21. Oud L. Epidemiology of Pregnancy-Associated ICU Utilization in Texas: 20012010. J Clin Med Res. 2017; 9(2): 143-53.

22. Lobato G MC, Reichenheim ME. Magnitude da depressão pós-parto no Brasil: uma revisão sistemática: Revista Brasileira de Saúde Materno Infantil. 2011; 11(4): 369-79.

23. Peraçoli JE PM. Síndromes hipertensivas na gestação: identificação de casos graves. Revista Brasileira de Ginecologia e Obstetrícia. 2005; 27(10): 627-34.

24. Main EK, Cape V, Abreo A, Vasher J, Woods A, Carpenter A, et al. Reduction of severe maternal morbidity from hemorrhage using a state perinatal quality collaborative. Am J Obstet Gynecol. 2017; 216(3): 298. e1-.e11.

25. Callaghan WM, Creanga AA, Kuklina EV. Severe maternal morbidity delivery and postpartum hospitalizations in the United States. Obstet Gynecol. 2012; 120(5): 1029-36. 